\title{
Analysis of thermal desorption of hydrogen in metallic alloys
}

\author{
A. Raina, V. S. Deshpande, N.A. Fleck \\ Cambridge University, Engineering Department, Trumpington St., Cambridge CB21PZ, UK \\ 21 August 2017
}

\begin{abstract}
The degree of embrittlement of metallic alloys is sensitive to the concentration of absorbed hydrogen, with hydrogen traps (particularly at grain boundaries) playing an important role. Thermal desorption spectrometry (TDS) is widely used to measure the detrapping and diffusion behaviour of hydrogen in metallic alloys. However, it is problematic to obtain a consistent interpretation of TDS data from the literature, due to the large number of material parameters that influence the measurement, and this results in a wide range of quoted values for trapping parameters such as the number of trap types, trap binding energies and trap densities. In this paper, the governing partial differential equation for hydrogen diffusion with sink and source terms for a single trap is formulated in non-dimensional form, assuming local equilibrium between the hydrogen atoms at the lattice sites and the trap sites. An asymptotic analysis reveals two distinct regimes of diffusion behaviour in TDS tests. Kissinger-type behaviour is expected in a TDS test of low heating rates on an alloy with a low lattice activation energy. Contour maps of maximum hydrogen flux and the corresponding temperature are plotted using axes of trap density and trap binding energy by making use of the full numerical solution (and asymptotic solutions). These maps serve as a useful tool for an accurate and simple determination of the trap binding energy as well as the trap density.
\end{abstract}

Keywords: Thermal desorption spectrometry, Hydrogen embrittlement, Diffusion, Trap binding energy, Trap density. 


\section{Introduction}

The pernicious effects of hydrogen on the mechanical properties of metallic alloys such as embrittlement are well known [1,2]. Microstructural defects such as dislocations, grain boundaries and vacancies strongly influence the solubility and rate of diffusion of hydrogen atoms in a metal-hydrogen system [3]. Thermal desorption spectrometry (TDS) and electropermeation (EP) experiments are widely used to measure the characteristics of hydrogen diffusion in metals. Recently, Raina et al. [4] presented an analysis of EP tests and mapped out the regimes of behaviour of hydrogen diffusion by both analytical and numerical calculations. Their design maps serve as a guideline for a unique determination of trap characteristics from EP test data. In the current study, we present the analysis of TDS tests based on the same theoretical background [4] in order to provide a unified interpretation of the physics of diffusion in both TDS and EP tests.

TDS tests are generally performed to determine the binding energy of hydrogen trap sites in metallic alloys [5]. In brief, the TDS test broadly consists of the following stages: charging of a sample with hydrogen via electrochemical or gaseous methods; heating the sample at a fixed rate, and detecting the flux of desorbing hydrogen atoms as a function of temperature by use of a mass spectrometer. The specimen is heated sufficiently slowly that no spatial gradients of temperature exist within it. This is straightforward to achieve as the thermal diffusivity of metallic alloys, on the order of $10^{-6} \mathrm{~m}^{2} / \mathrm{s}$, is much greater than the hydrogen lattice diffusion coefficient (on the order of $10^{-10} \mathrm{~m}^{2} / \mathrm{s}$ for ferritic steels).

Commonly, the evaluation of TDS test data is based on the first order rate theory of Kissinger [6]. Choo and Lee [7] used the Kissinger theory to evaluate the binding energy of traps in alpha iron from TDS tests, and subsequently this approach has been widely followed. However, the applicability of this method to study the diffusion of hydrogen in metals has been 
questioned due to the following shortcomings. First, the usual derivation of Kissinger's theory neglects diffusion. This implies that TDS data are independent of specimen thickness whereas experiments suggest that a thicker specimen leads to slower desorption [8]. Second, lattice diffusion of hydrogen is often accompanied by the trapping of hydrogen at microstructural defect sites [9] such as dislocations, grain boundaries and carbide particles. Kissinger's rate theory neglects the role of trapping mechanisms. And third, Kissinger's rate theory considers that hydrogen detrapping is the rate limiting step. Typically, the detrapping time is on the order of nanoseconds for trap sites of low binding energy, and this does not match the experimental diffusion times which are on the order of a few hundred seconds. In this paper, we build on the recent analysis of Kirchheim [13] and show that the Kissinger theory is a limiting case of the full diffusion equation with trapping and detrapping and is valid only for the restricted test regime of TDS tests performed at very slow heating rates on alloys with low lattice activation energy.

An accurate and physically motivated model for the diffusion of hydrogen in metals has been formulated by McNabb and Foster [10]. This theory assumes an energy landscape for the diffusion of hydrogen in metals of the form shown in Figure 1; McNabb and Foster account for the kinetics of trapping and detrapping from a single type of microstructural defect (trap) uniformly distributed in the metal. Coupled partial differential equations are derived in [10] for the concentrations of lattice and trapped hydrogen in space and time, and these require a numerical solution. The $\mathrm{McNabb}$ and Foster model requires rate constants for the trap kinetic equations, and the experimental determination of these is problematic. Hurley et al. [11] performed a parameter sensitivity study to the McNabb and Foster [10] theory, and emphasised that the predictions are sensitive to the choice of parameters such as the rate constants and the sample thickness. Oriani [12] simplified the analysis by showing that hydrogen atoms at lattice and trap sites can be considered to be in local equilibrium due to very fast trapping and 
detrapping. The local equilibrium condition allows one to replace the differential equation for trap kinetics by an algebraic relation between the lattice and trapped hydrogen concentrations. Kirchheim [13] validated the local equilibrium assumption [12] by performing Monte-Carlo simulations of 8 hydrogen atoms in 8000 octahedral sites of a fcc lattice. He also introduced a distribution of trap types in contrast to the single type of trap considered in $[10,12]$.

Several modified versions of McNabb and Foster [10] theory exist in order to model TDS test data. For example, Turnbull et al. [14] extended the McNabb and Foster theory to the case of two co-existing traps, deep and shallow traps, to model thermal desorption in metals. They showed that Kissinger-type analysis, as used by Choo and Lee [7], is only valid for very shallow traps with low concentration of hydrogen. Song et al. [15] assumed a different functional form for the trap kinetic equation to that of McNabb and Foster [10]; they performed TDS tests on a ferritic steel and showed good agreement of their numerical predictions with their data; and Ebihara et al. [16] solved the coupled differential equations of [10] by dropping the lattice diffusion term. Subsequently, Ebihara et al. [17] assumed a form of the lattice diffusion and local equilibrium relations that is strictly only valid in the limit of low trap occupancy. However, they assume trap enthalpies that are associated with a high trap occupancy. They compared their numerical predictions with the TDS data and showed poor agreement between their model assuming low trap occupancy (their Model II) and the available data. We conclude that their study highlights the need for a model that is able to include the role of diffusion and trapping/detrapping at high trap occupancies.

Recently, Kirchheim [18] has solved the diffusion equation [10] with traps in local equilibrium [12]. He considered the two cases of partially filled traps or fully saturated traps at the initial test temperature. Based on various simplifying assumptions, he obtained analytical solutions for the maximum flux and the corresponding temperature in a TDS test.

The remainder of this paper is organised as follows. In Section 2, we present the 
governing equations for the diffusion of hydrogen in TDS tests based on [10]; we consider a single trap type and assume local equilibrium [12]. The governing PDE is written in nondimensional form, and the dominant non-dimensional groups are identified. In Section 3, full numerical solutions are presented and contour maps are generated for the maximum flux and the corresponding temperature in the parametric space of trap binding energy and trap density. These contour maps serve as a useful graphical tool to extract the unknown trap binding energy and the trap density from a TDS test data. In Section 4, an asymptotic analytical approach reveals two distinct regimes I and II of diffusion behaviour: regime I assumes a low initial occupancy of traps whereas regime II assumes a high initial trap occupancy. A comparison of analytical and numerical solutions is presented, and the region of the map is identified for which Kissinger's theory is applicable. In Section 5, the effect of an initial rest period on the subsequent response to the TDS test is explored; this rest period represents the time at room temperature after hydrogen charging of the specimen, but prior to performing the TDS test. It is shown that the rest period can be highly significant. For example, hydrogen can quickly diffuse out of shallow traps and be released from the specimen prior to commencement of the TDS test.

\section{Theory of diffusion of hydrogen in metals}

\subsection{Hydrogen diffusion with source and sink term}

Hydrogen atoms occupy normal interstitial lattice sites (NILS) and additionally can reside at trapping sites such as interfaces or dislocations. The total hydrogen concentration $C$ is the sum of lattice hydrogen concentration $C_{L}$ and trapped hydrogen concentration $C_{T}$. Now, the lattice concentration $C_{L}(x, t)$ in space $x$ and time $t$ is dictated by Fickian diffusion over the NILS. However, in the presence of traps, hydrogen diffusion is modified by both trapping 
and detrapping of hydrogen atoms [10]. Mass conservation dictates that the rate of change of total concentration equals the net flux of diffusing hydrogen atoms $C_{L}(x, t)$, and this can be written in one-dimensional form as

$$
\frac{\partial C_{L}}{\partial t}+\frac{\partial C_{T}}{\partial t}=D_{L} \frac{\partial^{2} C_{L}}{\partial x^{2}} .
$$

Here, $D_{L}=D_{0} \exp (-Q / R T)$ is the lattice diffusion coefficient and is expressed in terms of the temperature $T$, lattice activation energy $Q$, diffusion pre-exponential factor $D_{0}$ and the universal gas constant $R$. Some typical values of the lattice activation energy $Q$ in metals and alloys are listed in Table 1. The full 3D version of (1) follows immediately by re-writing $\frac{\partial^{2} C_{L}}{\partial x^{2}}$ as $\nabla^{2} C_{L}$, but we shall limit our attention to the $1 \mathrm{D}$ case.

It is convenient to introduce the lattice and trap occupancy fractions $\theta_{L}$ and $\theta_{T}$, respectively, by re-writing the lattice and trap concentrations in the form $C_{L}=\theta_{L} \beta N_{L}$ and $C_{T}=\theta_{T} \alpha N_{T}$. Here, $\beta$ is the number of NILS per lattice atom, $N_{L}$ is the number of lattice atoms per unit volume, $\alpha$ is the number of atoms sites per trap and $N_{T}$ is the number of trap sites per unit volume. We emphasise that $0 \leq \theta_{L} \leq 1$ and $0 \leq \theta_{T} \leq 1$. Using these relations, equation (1) can be re-written as

$$
\frac{\partial \theta_{L}}{\partial t}+\left(\frac{\alpha N_{T}}{\beta N_{L}}\right) \frac{\partial \theta_{T}}{\partial t}=D_{L} \frac{\partial^{2} \theta_{L}}{\partial x^{2}}
$$

The net rate of trapped hydrogen concentration $\partial \theta_{T} / \partial t$ is obtained by considering the kinetics of trapping and detrapping, using standard rate theory [10]; the theory is summarised in the supplementary material, with the key result given by (A.4).

Oriani [12] assumed that a local equilibrium exists between the hydrogen atoms at the lattice sites and the trap sites, and is the limiting case of the trap kinetic equation (A.4) in the 
limit of a large atomic vibrational frequency. For the practical case where $\theta_{L} \ll 1$, we have

$$
\theta_{T}=\frac{K \theta_{L}}{1+K \theta_{L}}
$$

where the equilibrium constant $K$ is given in terms of the trap binding energy $\Delta H$ as

$$
K=\exp \left\{\frac{-\Delta H}{R T}\right\}
$$

Contours of trap occupancy fraction $\theta_{T}$ from (3) are shown in Figure 1b for selected values of the binding energy $\Delta H$ and for the choice $\theta_{L}=10^{-5}$. It shows the following features: (i) traps are full $\left(\theta_{T} \rightarrow 1\right)$ for $\Delta H \leq-40 \mathrm{~kJ} / \mathrm{mol}$ at room temperature, and (ii) trap occupancy approaches zero $\left(\theta_{T} \rightarrow 0\right)$ at a sufficiently high temperature $T$ for any given $\Delta H$ : this is the underlying principle behind the TDS test. Recall that, in TDS tests, the temperature $T$ at any time $t$ is defined in terms of the constant heating rate $\phi$ by

$$
T=T_{0}+\phi t
$$

where $T_{0}$ is the initial temperature, typically taken as $293 \mathrm{~K}$. The heating rate $\phi$ is sufficiently slow compared to the rate of thermal diffusion that the TDS specimen has a spatially uniform temperature $T(t)$.

\subsection{Governing diffusion equation with local equilibrium}

Assume local equilibrium between hydrogen atoms at the lattice and the trap sites, as given by

[3]. Then, upon making use of (3)-(5), the governing PDE for diffusion (2) can be re-cast in the simpler form

$$
\frac{\partial \theta_{L}}{\partial t}\left[1+\frac{\alpha N_{T} K}{\beta N_{L}\left(1+K \theta_{L}\right)^{2}}\right]+\frac{\alpha N_{T} K \Delta H \theta_{L} \phi}{\beta N_{L} R T^{2}\left(1+K \theta_{L}\right)^{2}}=D_{L} \frac{\partial^{2} \theta_{L}}{\partial x^{2}},
$$

with solution of functional form 


$$
\theta_{L}=\theta_{L}\left(x, t ; L, \theta_{L}^{0}, D_{0}, N_{T}, N_{L}, T_{0}, \phi, \Delta H, Q\right)
$$

The large number of independent parameters can lead to an intricate analysis of the TDS data. However, an identification of the governing non-dimensional groups leads to a much simpler statement of the governing PDE as follows.

\subsection{Non-dimensional groups and governing PDE}

Consider a one-dimensional specimen of length $L$ spanning the domain $-L / 2 \leq x \leq L / 2$. Introduce the following non-dimensional quantities: spatial coordinate $\bar{x} \equiv x / L$, time $\bar{t} \equiv t D_{0} / L^{2}$, heating rate $\bar{\phi} \equiv\left(\phi L^{2}\right) /\left(T_{0} D_{0}\right)$, trap density $\bar{N} \equiv\left(\alpha N_{T}\right) /\left(\beta N_{L}\right)$, temperature $\bar{T} \equiv T / T_{0}=1+\bar{\phi} \bar{t}$, lattice activation energy $\bar{Q} \equiv Q /\left(R T_{0}\right)$, trap binding energy $\overline{\Delta H} \equiv \Delta H /\left(R T_{0}\right)$, lattice diffusion coefficient $\bar{D}_{L} \equiv D_{L} / D_{0}=\exp (-\bar{Q} / \bar{T})$ and fractional lattice occupancy $\bar{\theta}_{L}$ such that $\bar{\theta}_{L}=\theta_{L} / \theta_{L}^{0}$. Then, the diffusion equation (2) with sink and source term becomes

$$
\frac{\partial \bar{\theta}_{L}}{\partial \bar{t}}+\left(\frac{\bar{N}}{\theta_{L}^{0}}\right) \frac{\partial \theta_{T}}{\partial \bar{t}}=\bar{D}_{L} \frac{\partial^{2} \bar{\theta}_{L}}{\partial \bar{x}^{2}}
$$

and equation (6) reduces to

$$
\frac{\partial \bar{\theta}_{L}}{\partial \bar{t}}\left[1+\frac{K \bar{N}}{\left(1+K \theta_{L}^{0} \bar{\theta}_{L}\right)^{2}}\right]+\frac{K \bar{N} \overline{\Delta H} \bar{\theta}_{L} \bar{\phi}}{\bar{T}^{2}\left(1+K \theta_{L}^{0} \bar{\theta}_{L}\right)^{2}}=\bar{D}_{L} \frac{\partial^{2} \bar{\theta}_{L}}{\partial \bar{x}^{2}}
$$

where $K$ in (4) can be re-expressed as $K=\exp (-\overline{\Delta H} / \bar{T})$. We note that (9) contains only 3 non-dimensional material parameters $(\bar{N}, \overline{\Delta H}, \bar{Q})$ and additionally 2 non-dimensional test parameters $\left(\theta_{L}^{0}, \bar{\phi}\right)$. In the remainder of our study we shall obtain full numerical solutions and approximate asymptotic solutions to (9). 


\subsection{Initial condition and boundary conditions for the TDS test}

Consider a one-dimensional TDS test on a specimen of length $L$ as shown schematically in Figure 2a. Initially, at time $t=0$, the specimen is at temperature $T_{0}$ with a spatially homogeneous distribution of lattice occupancy fraction $\theta_{L}=\theta_{L}^{0}$. Thereafter, the hydrogen lattice occupancy $\theta_{L}=0$ at the boundaries $x= \pm L / 2$ is maintained. In summary, the initial condition is $\bar{\theta}_{L}(\bar{x}, \bar{t}=0)=1$ and the boundary condition reads $\bar{\theta}_{L}(\bar{x}= \pm 1 / 2, \bar{t}>0)=0$.

Now increase the temperature of the specimen at a constant rate $\phi$. The lattice occupancy $\theta_{L}$ evolves spatially and temporally as sketched in Figure 2b. In a TDS test, the flux of hydrogen atoms $J(t)$ diffusing out at the boundaries $x= \pm L / 2$ is measured. Write $J(t)$ as the number of hydrogen atoms that exit the specimen per unit surface area, per unit time. This flux is related to the concentration gradient at the surface of the sample $(\bar{x}=1 / 2)$ such that

$$
J(t)=\left(\frac{-D_{L} \beta N_{L} \theta_{L}^{0}}{L}\right) \frac{\partial \bar{\theta}_{L}}{\partial \bar{x}} .
$$

A schematic of a typical TDS output is shown in Figure 2c. The total mass flow rate of atomic hydrogen $\dot{m}_{\mathrm{H}}$ from the specimen, of face area $2 \mathrm{~S}$, is given by

$$
\dot{m}_{\mathrm{H}}=\frac{2 J S M_{\mathrm{H}}}{N_{\mathrm{A}}},
$$

where $M_{\mathrm{H}}$ is the mass of one mole of atomic hydrogen and $N_{\mathrm{A}}$ is Avogadro's constant. Let $m_{\mathrm{S}}$ be the mass of the specimen, then the hydrogen desorption rate per unit mass of the specimen is given by

$$
\frac{\dot{m}_{\mathrm{H}}}{m_{S}}=\frac{2 J M_{\mathrm{H}}}{N_{\mathrm{A}} \rho L}
$$

where $\rho$ is the specimen density. Finally, a non-dimensional form of the total flux of 
desorbing hydrogen atoms is introduced as

$$
\bar{J}=\frac{2 J S t_{0}}{2 S L \beta N_{L}}
$$

where $t_{0}=L^{2} / D_{0}$ is the characteristic lattice diffusion time. Using (10), Eq. (13) reduces to

$$
\bar{J}=-\bar{D}_{L} \theta_{L}^{0} \frac{\partial \bar{\theta}_{L}}{\partial \bar{x}}
$$

In the following sections, we shall denote the maximum flux by $\bar{J}_{\max }$ and the corresponding temperature by $\bar{T}_{\max }$

\section{Numerical analysis of TDS tests}

The PDE (9) is solved numerically by using the partial differential equation solver pdepe in MATLAB $^{1}$. Consider a material representative of ferritic steel with lattice activation energy $Q=6.7 \mathrm{~kJ} / \mathrm{mol}$, diffusion pre-exponential factor $D_{0}=2 \times 10^{-7} \mathrm{~m}^{2} / \mathrm{s}$ and lattice density $N_{L}=8.46 \times 10^{28}$ atoms $\cdot \mathrm{m}^{-3}$ [9], with $\alpha=\beta=1$. Let the initial temperature be $T_{0}=293 \mathrm{~K}$ such that the non-dimensional lattice activation energy is $\bar{Q}=2.75$. In a typical TDS test, the initial concentration of hydrogen after electrochemical or gaseous charging is on the order of 0.02 wppm [15], implying an initial lattice occupancy fraction of $\theta_{L}^{0}=10^{-6}$. We choose a physically meaningful range of trap binding energy $-35 \leq \overline{\Delta H} \leq-10$, where the lower negative limit corresponds to $-24.3 \mathrm{~kJ} / \mathrm{mol}$ and the higher negative limit corresponds to $-85.3 \mathrm{~kJ} / \mathrm{mol}$, both at $T_{0}=293 \mathrm{~K}$. The trap density $N_{T}$ is taken to be in the range

\footnotetext{
1 The pdepe solver is based on the method of lines which converts the given PDE into a system of initial value problems. In this method, the spatial derivatives are replaced with algebraic approximations and the remaining time derivatives are solved as a system of ordinary differential equations. An automatic time-stepping routine in pdepe solver ensures temporal convergence is achieved in each solution step. All simulations used a uniform mesh with element size $e=0.005 L$. The chosen element size was sufficient for all the regimes to obtain mesh independent results.
} 
$\left(10^{-2}-10^{-7}\right) N_{L}$, hence $10^{-7} \leq \bar{N} \leq 10^{-2}$ [12]. The heating rate is varied from $\bar{\phi}=0.01$ to 1.0 , which corresponds to $\phi=0.012 \mathrm{~K} / \mathrm{s}$ to $0.12 \mathrm{~K} / \mathrm{s}$, respectively, for a specimen of thickness $L=5 \times 10^{-3} \mathrm{~m}$ assuming the given values of $T_{0}$ and $D_{0}$.

\subsection{Contour maps of $\bar{J}_{\max }$ and $\bar{T}_{\max }$}

Consider a typical TDS test, as shown in Figure 2. In general, the values of lattice activation energy $\bar{Q}$ and heating rate $\bar{\phi}$ are known a priori. A numerical simulation provides $\bar{J}(\bar{t})$ for the assumed $(\bar{Q}, \bar{\phi})$, and for any given values of $\left(\theta_{L}^{0}, \bar{N}, \overline{\Delta H}\right)$. The response $\bar{J}(\bar{t})$ is converted directly to $\bar{J}(\bar{T})$ by making use of the linear relation $\bar{T}=1+\bar{\phi} \bar{t}$.

We proceed to propose the practical problem: what is the best procedure for deducing the values of $\left(\theta_{L}^{0}, \bar{N}, \overline{\Delta H}\right)$ from a given $\bar{J}(\bar{T})$ curve? The initial lattice occupancy $\theta_{L}^{0}$ is deduced from the area under the $\bar{J}(\bar{T})$ curve of data. This leaves the trap binding energy $\overline{\Delta H}$ and trap density $\bar{N}$ as primary unknowns. Now make use of multiple numerical solutions of the PDE (9) in order to construct contours of maximum flux $\bar{J}_{\max }$, and the corresponding temperature $\bar{T}_{\max }$, on a map with axes $\bar{N}$ and $\overline{\Delta H}$, for selected values of $\bar{\phi}$ , and for the choice $\bar{Q}=2.75$ and $\theta_{L}^{0}=10^{-6}$. The resulting contours of $\bar{J}_{\max } / \bar{\phi}$ are plotted in Figure 3a: we adopt the normalisation $\bar{J}_{\max } / \bar{\phi}$ as numerical experimentation has revealed that $\bar{J}_{\max }$ scales almost linearly with $\bar{\phi}$. Several characteristic features emerge from this map. First, a zone exists in the lower, right quadrant for which no peak flux is attained. The boundary of this zone is given in Figure 3a for selected values of heating rate $\bar{\phi}$. Second, a transition in desorption behaviour occurs at $\overline{\Delta H} \approx-14$. Shallow traps exist for $\overline{\Delta H}>-14$, 
and in this regime the normalised peak flux $\bar{J}_{\max } / \bar{\phi}$ is sensitive to the choice of both $\bar{N}$ and $\overline{\Delta H}$; we shall refer to this as regime I. In contrast, deep traps exist for $\overline{\Delta H}<-14$ and, in this so-called regime II, $\bar{J}_{\max } / \bar{\phi}$ is sensitive to the choice of $\bar{N}$ but not of $\overline{\Delta H}$. Contours of $\bar{T}_{\max }$ are shown for selected heating rates $\bar{\phi}$ in Figure $3 \mathrm{~b}$. The switch in regimes here is apparent from a minor slope change at $\overline{\Delta H} \approx-14$. Although the contour maps have only been presented for the choice $\theta_{L}^{0}=10^{-6}$, we find that changing the value of $\theta_{L}^{0}$ by an order of magnitude has a negligible effect upon several of the results. For example, if $\theta_{L}^{0}=10^{-5}$, the transition from regime I to regime II occurs at $\overline{\Delta H} \approx-12$. Also, upon increasing $\bar{Q}$, the contours of $\bar{J}_{\max } / \bar{\phi}$ change only slightly, whereas the contours of $\bar{T}_{\max }$ shift significantly; this sensitivity analysis is not shown explicitly for the sake of conciseness. Additional insight is gained into the nature of regimes I and II in the following section by the generation of approximate solutions to the governing PDE.

The contours maps provide us with a graphical tool for the identification of the parameters $\overline{\Delta H}$ and $\bar{N}$ from $\bar{J}_{\max } / \bar{\phi}$ and $\bar{T}_{\max }$ TDS data. But now a word of caution: within regime I, the contours of maximum flux and the corresponding temperature are nearly parallel. We conclude that, in regime I, a unique determination of either the trap binding energy or the trap density can be achieved provided one of the two quantities is known a priori.

\section{Asymptotic analysis of the TDS test}

The PDE (9) is reduced to an approximate ordinary differential equation, ODE, and associated approximate solutions in this section. We begin by assuming that an approximate, separation of variables solution is of the form [18]

$$
\bar{\theta}_{L}(\bar{x}, \bar{t})=f(\bar{t}) \cos (\pi \bar{x}) .
$$


This satisfies the boundary conditions at $\bar{x}= \pm 1 / 2$, and the initial condition is satisfied on the mid-plane of the specimen $(\bar{x}=0)$ by taking $f(\bar{t}=0)=1$. Upon substitution of (15) into the PDE (9) and by focussing our attention on $\bar{x}=0$, a non-linear ordinary differential equation (ODE) in $\bar{t}$ follows immediately as

$$
\frac{d f}{d \bar{t}}\left[1+\frac{K \bar{N}}{\left(1+K \theta_{L}^{0} f\right)^{2}}\right]+\frac{K \bar{N} \overline{\Delta H} \bar{\phi} f}{\bar{T}^{2}\left(1+K \theta_{L}^{0} f\right)^{2}}=-\pi^{2} \bar{D}_{L} f .
$$

Once we have solved (16) for $f(\bar{t})$ back substitution into (15) gives an approximate solution for $\bar{\theta}_{L}(\bar{x}, \bar{t})$.

The non-dimensional flux $\bar{J}$ at $\bar{x}=1 / 2$ can be expressed in terms of $f$ as $\bar{J}=\pi \bar{D}_{L} \theta_{L}^{0} f$ and its maximum value as $\bar{J}_{\max }=\pi \bar{D}_{L} \theta_{L}^{0} f_{\max }$. In a TDS test, the maximum flux is attained when $\partial \bar{J} / \partial \bar{t}=0$. After substitution of (15) in (14), this condition can be reexpressed as

$$
\frac{d f}{d \bar{t}}=-\left(\frac{\bar{Q} \bar{\phi}}{\bar{T}^{2}}\right) f \quad \text { at } \quad \bar{J}=\bar{J}_{\max }
$$

Write $f=f_{\max }$ at $\bar{J}=\bar{J}_{\max }$; then, upon substituting (17) into (16), we obtain an algebraic relation between $f_{\max }$ and $\bar{T}_{\max }$,

$$
-\bar{Q} \bar{\phi}\left[1+\frac{K \bar{N}}{\left(1+K \theta_{L}^{0} f_{\max }\right)^{2}}\right]+\frac{K \bar{N} \overline{\Delta H} \bar{\phi}}{\left(1+K \theta_{L}^{0} f_{\max }\right)^{2}}+\pi^{2} \bar{D}_{L} \bar{T}_{\max }^{2}=0
$$

where $K=K\left(\bar{T}_{\text {max }}\right)$ and $\bar{D}_{L}=\bar{D}_{L}\left(\bar{T}_{\max }\right)$. Now proceed to obtain approximate solutions to the algebraic equation (18) for $\bar{T}_{\max }$ by considering the two physical limits of diffusion in a TDS test: the shallow trap regime I of $K \theta_{L}^{0} \ll 1$ and the deep trap regime II of $K \theta_{L}^{0} \gg 1$. 


\subsection{Shallow traps (Regime I)}

In the shallow trap regime $\mathrm{I}$, we have $K \theta_{L}^{0} \ll 1$ at the initial temperature $\bar{T}=1$. Full numerical simulations reveal that $f$ increases slightly (to a maximum value of less than 4 ) before decreasing to zero. Hence, we can assume that $K \theta_{L}^{0} f_{\max } \ll 1$, and equation (18) reduces to

$$
\ln \left(\frac{\bar{\phi}}{\bar{T}_{\max }^{2}}\right)=\frac{\overline{\Delta H}-\bar{Q}}{\bar{T}_{\max }}+\ln \left(\frac{K \pi^{2}}{\bar{Q}-K \bar{N}(\overline{\Delta H}-\bar{Q})}\right) .
$$

Differentiation of (19) with respect to $1 / \bar{T}_{\max }$ gives

$$
\frac{\partial \ln \left(\bar{\phi} / \bar{T}_{\max }^{2}\right)}{\partial\left(1 / \bar{T}_{\max }\right)}=(\overline{\Delta H}-\bar{Q})-\frac{\bar{Q} \overline{\Delta H}}{\bar{Q}-K \bar{N}(\overline{\Delta H}-\bar{Q})} .
$$

Now, Kissinger's theory [6] states that the right-hand side of (20) equals $\overline{\Delta H}-\bar{Q}$; the degree to which this condition is satisfied is addressed below, after a similar analysis is developed for deep traps.

\subsection{Deep traps (Regime II)}

Consider the deep trap limit $K \theta_{L}^{0} \gg 1$ at the initial temperature $\bar{T}=1$, such that $\theta_{T} \approx 1$ initially, as demanded by (3). Kirchheim [18] assumed that such traps are half-filled $\left(\theta_{T}=0.5\right)$ at $\overline{\boldsymbol{J}}=\overline{\boldsymbol{J}}_{\text {max }}$. Upon adopting this criterion, we assume that $K \theta_{L}^{0} f_{\max }=1$ in (18) to obtain an implicit relation for $\bar{T}_{\max }$ in the form

$$
\ln \left(\frac{\bar{\phi}}{\bar{T}_{\max }^{2}}\right)=\frac{\overline{\Delta H}-\bar{Q}}{\bar{T}_{\max }}+\ln \left(\frac{4 K \pi^{2}}{4 \bar{Q}-K \bar{N}(\overline{\Delta H}-\bar{Q})}\right)
$$

Differentiation of (21) with respect to $1 / \bar{T}_{\max }$ yields

$$
\frac{\partial \ln \left(\bar{\phi} / \bar{T}_{\max }^{2}\right)}{\partial\left(1 / \bar{T}_{\max }\right)}=(\overline{\Delta H}-\bar{Q})-\frac{4 \bar{Q} \overline{\Delta H}}{4 \bar{Q}-K \bar{N}(\overline{\Delta H}-\bar{Q})} .
$$


Again, it is clear that the right hand side deviates from the value $\overline{\Delta H}-\bar{Q}$ of Kissinger's theory [6]; the extent of this deviation is explored in the sequel. The maximum flux $\bar{J}_{\text {max }}$ follows immediately from the assumption $K \theta_{L}^{0} f_{\max }=1$, to give

$$
\bar{J}_{\max }=\pi \frac{\bar{D}_{L}\left(\bar{T}_{\max }\right)}{K\left(\bar{T}_{\max }\right)} .
$$

\subsection{Comparison of numerical and asymptotic solutions of $\bar{T}_{\max }$}

A comparison of the asymptotic and numerical predictions are given in Figure 4 for both regimes I and II: the asymptotic solutions for $\bar{T}_{\max }$ are given by (19) and (21) in regimes I and II, respectively. Excellent agreement is noted between numerical and asymptotic solutions.

\subsection{Scope of validity of Kissinger theory in regimes I and II:}

According to the usual Kissinger theory [6], the slope of the $\ln \left(\bar{\phi} / \bar{T}_{\max }^{2}\right)$ versus $1 / \bar{T}_{\max }$ plot is of the form

$$
\frac{\partial \ln \left(\bar{\phi} / \bar{T}_{\max }^{2}\right)}{\partial\left(1 / \bar{T}_{\max }\right)}=\overline{\Delta H}-\bar{Q} .
$$

What is the relationship between this equation and relations (20) and (22) for regimes I and II, respectively? Let $\epsilon$ be the fractional deviation of the right-hand side of (20) from the righthand side of (24), for regime I, such that

$$
\frac{\bar{Q} \overline{\Delta H}}{\bar{Q}-K \bar{N}(\overline{\Delta H}-\bar{Q})}=\epsilon(\overline{\Delta H}-\bar{Q})
$$

This condition can be rearranged to the form

$$
K \bar{N}=\frac{1}{\xi+1}\left(-1+\frac{\xi}{\epsilon(\xi+1)}\right),
$$

where $\xi \equiv-\overline{\Delta H} / \bar{Q}$ and $K=K\left(\bar{T}_{\max }\right)$. It has the interpretation that, for this value of $K \bar{N}$, 
Kissinger's theory conforms to the more accurate solution (20) to within a deviation of $\epsilon$; the deviation is less for greater value of $K \bar{N}$. For convenience of plotting contours of (26), we introduce the normalisation

$$
\Psi_{\mathrm{I}}=\frac{K \bar{N}}{\frac{1}{\xi+1}\left(-1+\frac{\xi}{\epsilon(\xi+1)}\right)}
$$

and note that for $\Psi_{\mathrm{I}} \geq 1$, Kissinger theory conforms to the more accurate solution (20) to within a deviation of $\epsilon$. The following corollary holds, and is of more practical use. Assume that $\bar{N}$ is known. Then, the trap binding energy $\overline{\Delta H}$, as predicted by Kissinger's theory, is accurate to within a small assumed error $\epsilon$ provided the TDS test lies within the regime $\Psi_{\mathrm{I}} \geq 1$

Likewise, write $\epsilon$ as the fractional deviation of the right-hand side of (22) from the right-hand side of (24), for regime II. Then, the condition for Kissinger's theory to be valid in regime II can be written as $\Psi_{\mathrm{II}} \geq 1$ where

$$
\Psi_{\mathrm{II}}=\frac{K \bar{N}}{\frac{4}{\xi+1}\left(-1+\frac{\xi}{\epsilon(\xi+1)}\right)} .
$$

\subsubsection{Map of regimes where Kissinger's theory holds}

In order to plot the contours of $\Psi_{\mathrm{I}}=1$ and $\Psi_{\mathrm{II}}=1$ on the regime map with axes of $\bar{N}$ and $\overline{\Delta H}$, PDE (9) needs to be solved numerically to first obtain contours of $\bar{T}_{\text {max }}$, as done in Figure 3b. A regime map which shows the contours of $\Psi_{I}=1$ and $\Psi_{I I}=1$ corresponding to the material parameters used in Figure $3 \mathrm{~b}$ is plotted in Figure 5a for a relative error $\epsilon=0.1$

. The role of $\bar{Q}$ is also explored, by increasing its value from 2.75 in Figure 5a to 12.75 in Figure 5b. For $\bar{\phi}=0.01$, we find that both $\Psi_{\mathrm{I}}>1$ and $\Psi_{\mathrm{II}}>1$ throughout the map: at this 
low heating rate, Kissinger's theory always holds. In contrast, for $\bar{\phi}=0.1$, Kissinger theory holds in most of regimes I and II; and for $\bar{\phi}=1.0$, Kissinger theory holds only in a small portion of regimes I and II.

We repeat the analysis for $\bar{Q}=12.75$, which is representative of pure nickel, keeping all other parameters same as before. The results are plotted in Figure 5b. A maximum flux is always obtained for this case. We note that $\Psi_{\mathrm{I}} \ll 1$ in regime $\mathrm{I}$ for all assumed heating rates, hence, Kissinger's theory does not hold in regime I. In regime II, contours of $\Psi_{\mathrm{II}}=1$ are shown for $\bar{\phi}=0.01$ and 0.1 , above which Kissinger's theory holds (with a relative error of $\epsilon=0.1$ or less). For $\bar{\phi}=1.0, \Psi_{\mathrm{II}} \ll 1$ in the entire regime II and hence Kissinger's theory does not hold.

\section{Effect of rest period on TDS tests}

TDS tests often involve holding a specimen at room temperature for a rest period $t_{\text {rest }}$ between charging and testing. To explore the effect of rest time at room temperature, (5) is modified to

$$
T=T_{0}+\phi\left\langle t-t_{\text {rest }}\right\rangle
$$

where $\langle\cdot\rangle$ represents the Macaulay brackets such that $\langle x\rangle=x$ if $x \geq 0$, otherwise $\langle x\rangle=0$.

The non-dimensional resting time becomes $\bar{t}_{\text {rest }} \equiv\left(D_{0} t_{\text {rest }}\right) / L^{2}$ and the modified nondimensional temperature $\bar{T}$ reads

$$
\bar{T}=1+\bar{\phi}\left\langle\bar{t}-\bar{t}_{\text {rest }}\right\rangle
$$

Simulations with PDE (9) are performed at rest period $\bar{t}_{\text {rest }}=[0,0.25,0.5,1]$ and $\bar{\phi}=0.1$ for regime I ( $\overline{\Delta H}=-8)$. The remaining parameters are unchanged from those used in Section 3.1. The trap occupancy fraction $\theta_{T}$ is plotted in Figure 6 a as a function of position $\bar{x}$ at the 
end of the rest period. (The initial trap occupancy fraction $\theta_{T}^{0}$ is obtained from $\theta_{L}^{0}$ via (3).) The effect of rest period on the flux $\bar{J}$ versus $\bar{T}$ response is shown in Figure 6b: the initial spike due to rapid desorption of lattice hydrogen, and the maximum flux both drop with increasing $\bar{t}_{\text {rest }}$. We observe that a specimen with lower trap binding energy $(\overline{\Delta H}=-8)$ is completely depleted of hydrogen in approximately two hours rest time, whereas a specimen with higher trap binding energy $(\overline{\Delta H}=-20)$ takes several weeks to lose its hydrogen content.

\section{Conclusions}

This paper presents a non-dimensional partial differential equation (PDE) with reduced number of independent parameters for the analysis of thermal desorption spectrometry (TDS) tests. The governing PDE is obtained from the trap kinetics model of McNabb \& Foster (1963) by incorporating the local equilibrium condition of Oriani (1970). Asymptotic analysis of the governing PDE reveals two distinct regimes of diffusion: regime I is associated with shallow traps whereas regime II is associated with deep traps. These are validated by full numerical solutions of the governing PDE. Kissinger's theory is a limiting case of the asymptotic solutions and conditions are identified for which it is accurate to within a stated error. Based on full numerical solutions of the governing PDE, contour maps of non-dimensional peak flux and the corresponding temperature are presented for materials representative of ferritic steels. The contour maps serve as a tool for the graphical determination of trap binding energy and trap density from given TDS data. In regime I, either trap density or trap binding energy must be known for the unique determination of the remaining unknown parameter. In regime II, both the binding energy and trap density can be determined. The effect of a rest period at room temperature prior to starting the TDS test is presented; significant loss of hydrogen can occur during this rest period. 


\section{Acknowledgement}

The authors would like to acknowledge the funding and technical support from BP through the BP International Centre for Advanced Materials (BP-ICAM) which made this research possible.

\section{References}

[1] Gangloff R. P. and Somerday B. P., Gaseous hydrogen embrittlement of materials in energy technolgies, 1st Edition: The problem, its characterisation and effects on particular alloy classes (2012) USA: Woodhead Publishing.

[2] Bhadeshia H. K. D. H, Prevention of hydrogen embrittlement in steels. ISIJ International, 56 (2016) 24-36.

[3] Pundt A. and Kirchheim R., Hydrogen in metals: microstructural aspects. Annual Review of Materials Research 36 (2006) 555-608.

[4] Raina A., Deshpande V. S. and Fleck N. A., Analysis of electro-permeation of hydrogen in metallic alloys. Philosophical Transactions of the Royal Society A 375 (2017) 20160409.

[5] Young G. A. Jr. and Scully J. R., The diffusion and trapping of hydrogen in high purity aluminum, Acta Materialia 46 (1998) 6337-6349.

[6] Kissinger H. E., Variation of peak temperature with heating rate in differential thermal analysis. Journal of Research of the National Bureau of Standards 57(4) (1956) 217-221.

[7] Choo W. Y. and and Lee J. Y., Thermal analysis of trapped hydrogen in pure iron. Metallurgical Transactions 13A (1982) 135-140.

[8] Enomoto M. and Hirakami D., Influence of specimen thickness on thermal desorption spectrum of hydrogen in high strength SCN435 Steel. ISIJ International 55(11) (2015) 2492-2498. 
[9] Novak P., Yuan, R., Somerday, B. P., Sofronis, P. and Ritchie, R. O. A statistical, physicalbased, micro-mechanical model of hydrogen-induced intergranular fracture in steel. Journal of the mechanics and physics of solids 58 (2010) 206-226.

[10] McNabb A. and Foster P. K., A new analysis of the diffusion of hydrogen in iron and ferritic steels. Transactions of the Metallurgical Society of AIME 227 (1963) 618-627.

[11] Hurley C., Martin F., Marchetti L., Chêne J., Blanc C. and Andrieu E., Numerical modeling of thermal desorption mass spectroscopy (TDS) for the study of hydrogen diffusion and trapping interactions in metals. Int. J. Hydrogen Energy 40 (2015) 34023414 .

[12] Oriani R. A. The diffusion and trapping of hydrogen in steel. Acta Metallurgica 18 (1970) 147-157.

[13] Kirchheim R., Hydrogen solubility and diffusibilty in defective and amorphous metals. Progess in Materials Science 32 (1988) 261-325.

[14] Turnbull A., Hutchings R. B. and Ferriss D.H., Modelling of thermal desorption of hydrogen from metals. Materials Science and Engineering A238 (1997) 317-328.

[15] Song E. J., Suh D. W. and Bhadeshia H. K. D. H., Theory for hydrogen desorption in ferritic steel. Computational Material Science 79 (2013) 36-44.

[16] Ebihara K., Suzudo T., Kaburaki H., Takai K. and Takebayashi S., Modeling of hydrogen thermal desorption profile of pure iron and eutectoid steel. ISIJ International 47(8) (2007) 1131-1140.

[17] Ebihara K., Kaburaki H., Suzudo T. and Takai K., A numerical study on the validity of the local equilibrium hypothesis in modeling hydrogen thermal desorption spectra. ISIJ International 49(12) (2009) 1907-1913.

[18] Kirchheim R., Bulk diffusion-controlled thermal desorption spectroscopy with examples for hydrogen in iron. Metallurgical and Materials Transactions A, 47(A) (2016) 672-696. 
[19] Ono K. and Meshii M. Hydrogen detrapping from grain boundaries and dislocations in high purity iron. Acta Metallurgica et Materialia, 40(6) (1992) 1357-1364.

[20] Li D., Gangloff R. P. and Scully, J. R., Hydrogen trap states in ultrahigh-strength AERMET 100 Steel. Metallurgical and Materials Transactions A 35(A) (2004) 849-864.

[21] Kimura A. and Birnbaum H. K., Hydrogen induced grain boundary fracture in high purity nickel and its alloys-Enhanced hydrogen diffusion along grain boundaries. Acta Metallurgica, 36(3) (1988) 757-766.

[22] Katz L., Guinan M. J. and Borg R. Diffusion of $\mathrm{H}_{2}, \mathrm{D}_{2}$, and $\mathrm{T}_{2}$ in single-crystal Ni and Cu. Physical Review B 4(2) (1971) 330-341.

[23] Zhou X. W., Gabaly F. E., Stavila V. and Allendorf M. D. Molecular dynamics simulations of hydrogen diffusion in aluminum. Journal of Physical Chemistry C 120 (2016) 75007509.

[24] Brass A. M. and Chêne J., Hydrogen uptake in 316L stainless steel: Consequences on the tensile properties. Corrosion Science 48 (2006) 3222-3242.

\section{Table}

\begin{tabular}{|l|l|l|l|l|}
\hline Metal/Alloy & $Q[\mathrm{~kJ} / \mathrm{mol}]$ & $D_{0}\left[\mathrm{~m}^{2} / \mathrm{s}\right]$ & $D_{L}\left[\mathrm{~m}^{2} / \mathrm{s}\right]$ & Reference \\
\hline Pure iron (ferritic) & 6.7 & $2 \times 10^{-7}$ & $1.2 \times 10^{-8}$ & Ono \& Meshii [19] \\
AerMet100 (martensitic) & 10.8 & $7.1 \times 10^{-10}$ & $8.43 \times 10^{-12}$ & Li et al. [20] \\
Pure nickel & 33.3 & $2.5 \times 10^{-7}$ & $3 \times 10^{-13}$ & Kimura \& Birnbaum [21] \\
Pure copper & 38.8 & $1.13 \times 10^{-6}$ & $1.32 \times 10^{-13}$ & Katz et al. [22] \\
Pure aluminium & 39.6 & $1.9 \times 10^{-5}$ & $1.68 \times 10^{-12}$ & Zhou et al. [23] \\
SAE 316L (austenitic) & 53.6 & $6.2 \times 10^{-7}$ & $1.72 \times 10^{-16}$ & Brass \& Chêne [24] \\
\hline
\end{tabular}

Table 1: Data of lattice activation energy $Q$, diffusion pre-exponential factor $D_{0}$ and lattice diffusion coefficient $D_{L}=D_{0} \exp (-Q / R T)$ at $T=293 \mathrm{~K}$ for the diffusion of hydrogen atoms in metals and alloys. 


\section{Figures}

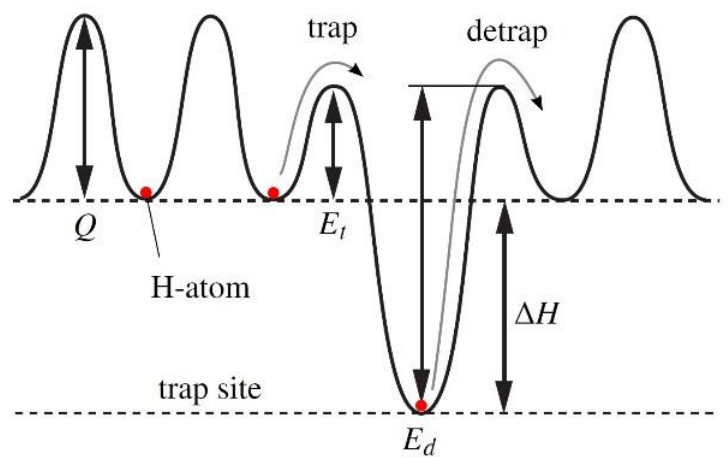

(a)

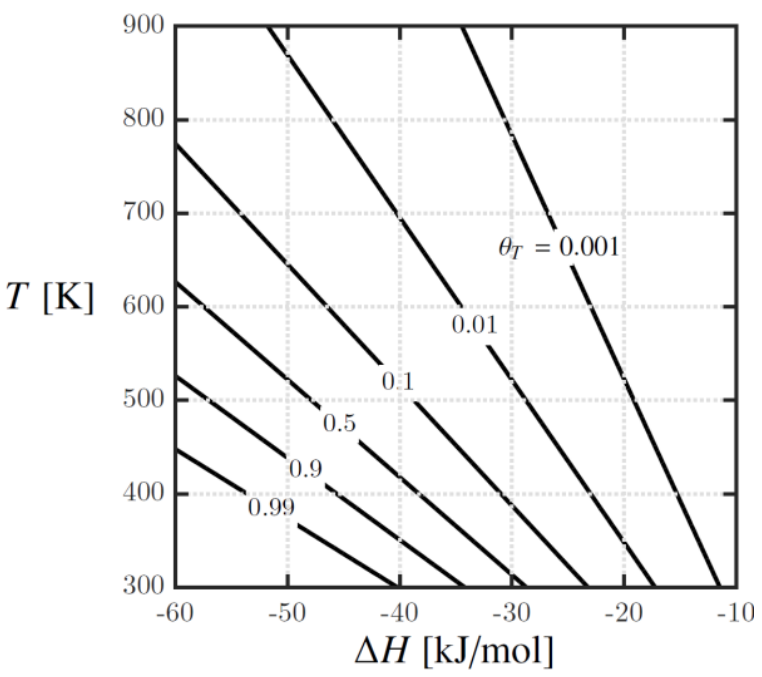

(b)

Figure 1: (a) A schematic of different energy levels for diffusion of hydrogen in metals. Energy levels lower than interstitial lattice activation energy $Q$ are referred to as traps, which can be classified as shallow or deep. $\Delta H(<0)$ is the trap binding energy, $E_{t}(>0)$ is the trapping activation energy and $E_{d}(>0)$ is the detrapping activation energy. (b) Contours of trap occupancy fraction $\theta_{T}$ using equation (3) are plotted on the axes of trap binding energy $\Delta H$ and temperature $T$ at lattice occupancy $\theta_{L}=10^{-5}$.

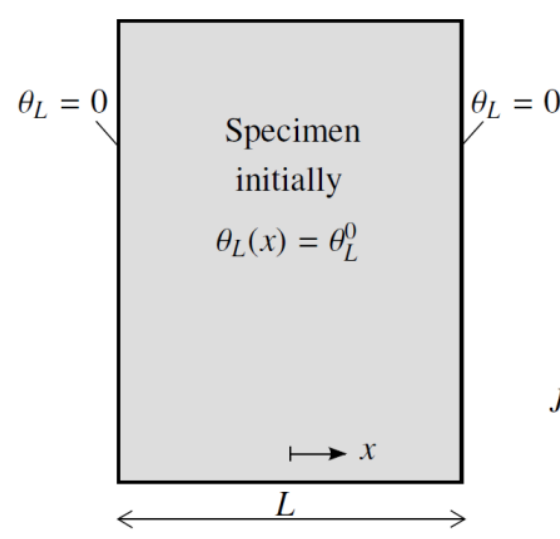

(a)

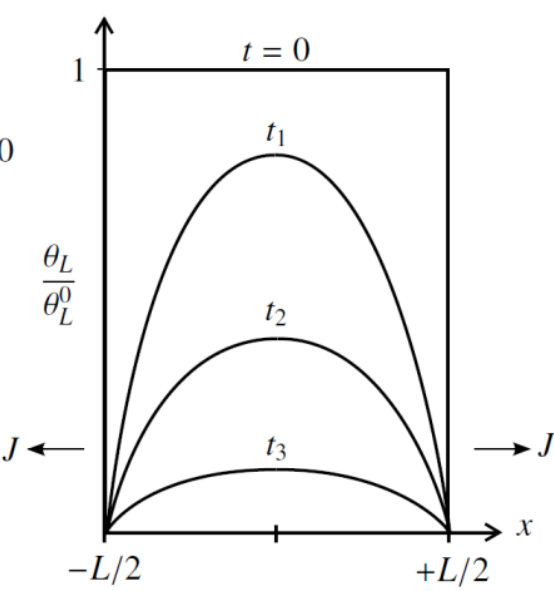

(b)

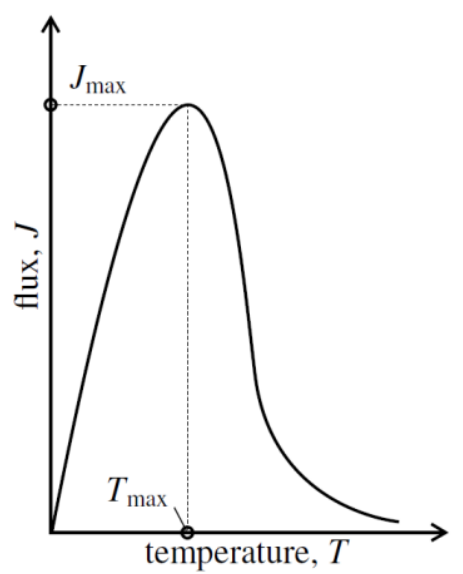

(c)

Figure 2: (a) A schematic illustration of initial condition and boundary conditions in a TDS test. (b) Transient solution curves of the normalised lattice occupancy fraction $\theta_{L} / \theta_{L}^{0}$ at different times $0<t_{1}<t_{2}<t_{3}$ along the specimen thickness $L$. (c) A schematic of typical TDS output data in the form of hydrogen desorption flux versus temperature. Temperature $T_{\max }$ where maximum flux $J_{\max }$ is obtained are marked. 


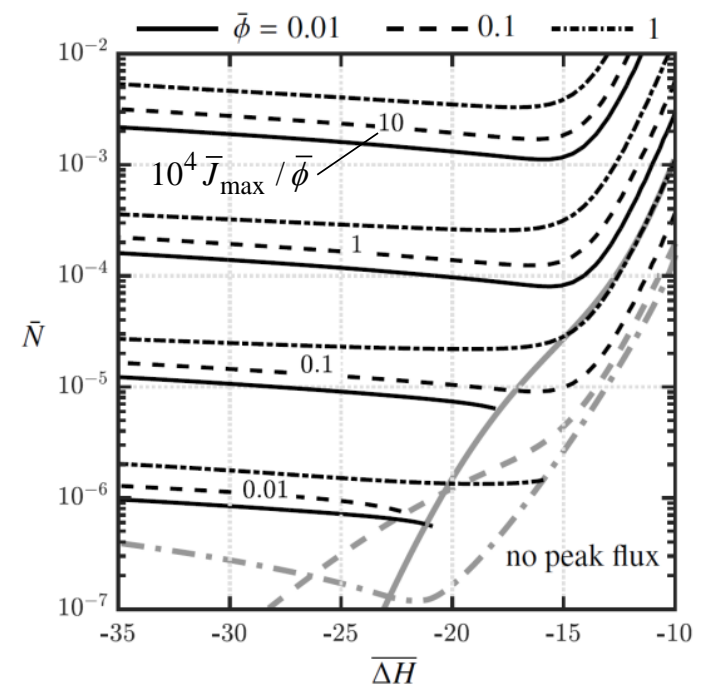

(a)

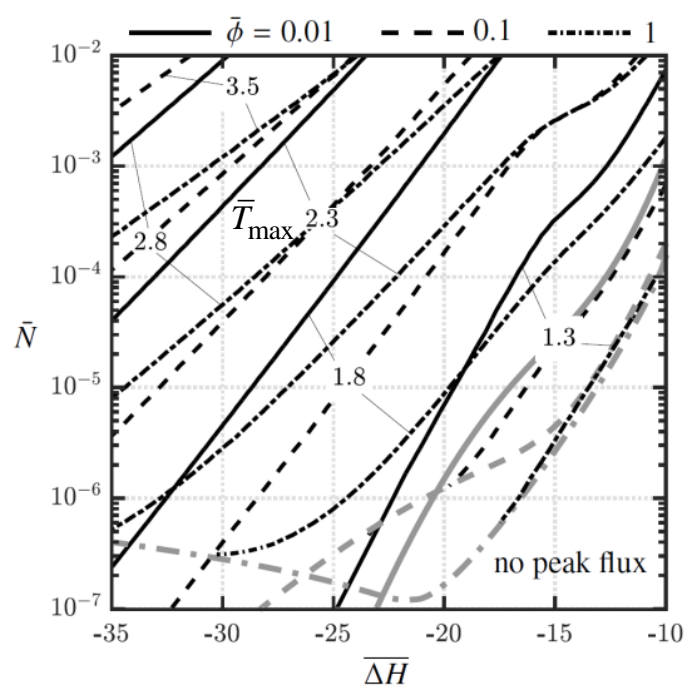

(b)

Figure 3: Contours of (a) scaled and normalised maximum flux $10^{4} \bar{J}_{\max } / \bar{\phi}$ and (b) temperature $\bar{T}_{\max }$, obtained by numerically solving PDE (9) are plotted on the axes of $\bar{N}$ and $\overline{\Delta H}$. Parameters $\theta_{L}^{0}=10^{-6}$ and $\bar{Q}=2.75$ are used with different $\bar{\phi}$. The curves (dark gray) separating the bottom right corner from flux contours represents the region where no peak flux is observed. These curves are plotted for each $\bar{\phi}$ using the same line style.

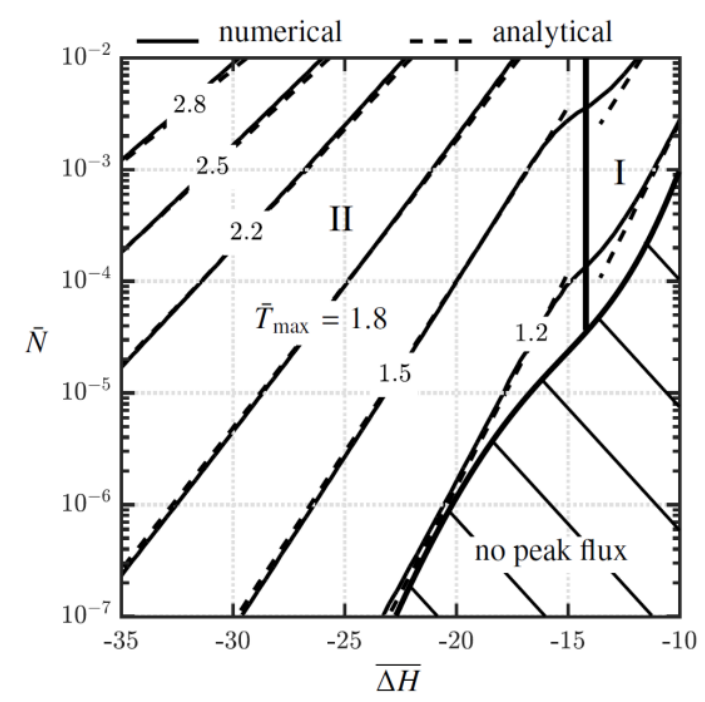

(a)

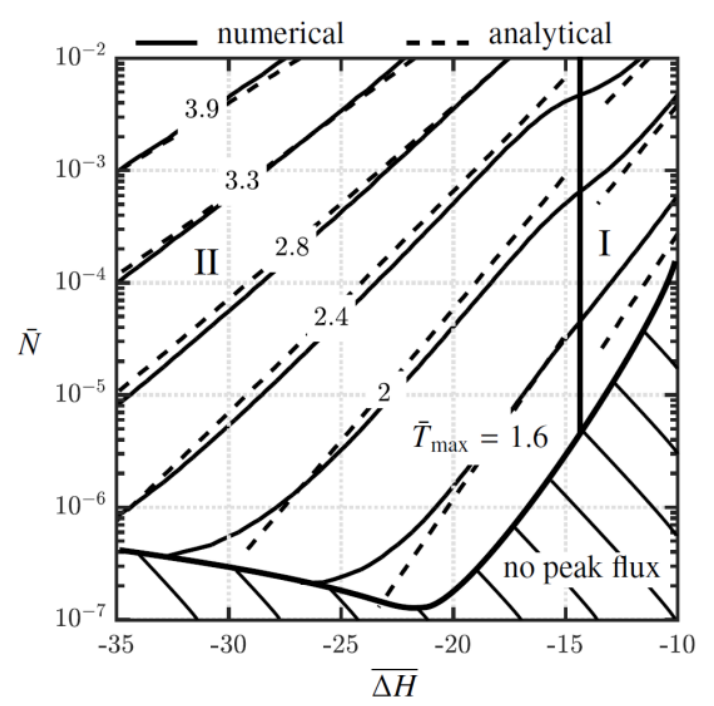

(b)

Figure 4: Comparison of analytical solutions (19) and (21) of temperature $\bar{T}_{\max }$ in regimes I and II, respectively, with full numerical solution of (9). Contours are plotted over the parametric space of $\bar{N}$ and $\overline{\Delta H}$ for (a) $\bar{\phi}=0.01$ and (b) $\bar{\phi}=1.0$ at $\theta_{L}^{0}=10^{-6}$ and $\bar{Q}=2.75$. Transition between regimes I and II takes place at $\overline{\Delta H} \approx-14$ where asymptotic solutions do not hold. Regions of no peak flux are also indicated. 


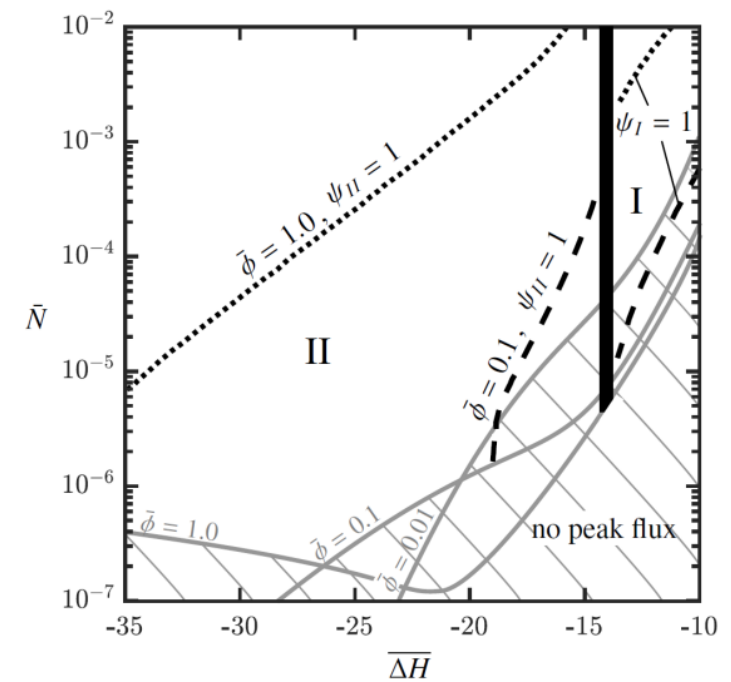

(a)

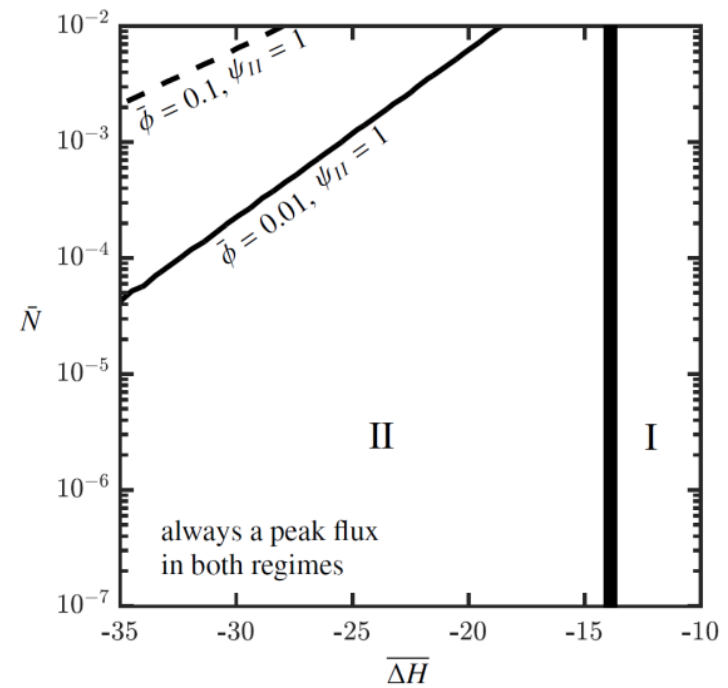

(b)

Figure 5: The regions on regimes map where Kissinger theory holds are shown by plotting contours of $\Psi_{\mathrm{I}}=1$ (27) and $\Psi_{\mathrm{II}}=1$ (28) for (a) $\bar{Q}=2.75$ and (b) $\bar{Q}=12.75$ at $\bar{\phi}=0.01$, 0.1 and 1.0. Kissinger's theory holds in regions above $\Psi_{\mathrm{I}}=\Psi_{\mathrm{II}}=1$ at given $\bar{\phi}$ with a relative error $\epsilon=0.1$ or less. The region of no peak flux is shown by shaded lines in (a) for different $\bar{\phi}$, whereas in (b) there is always a unique maximum flux.

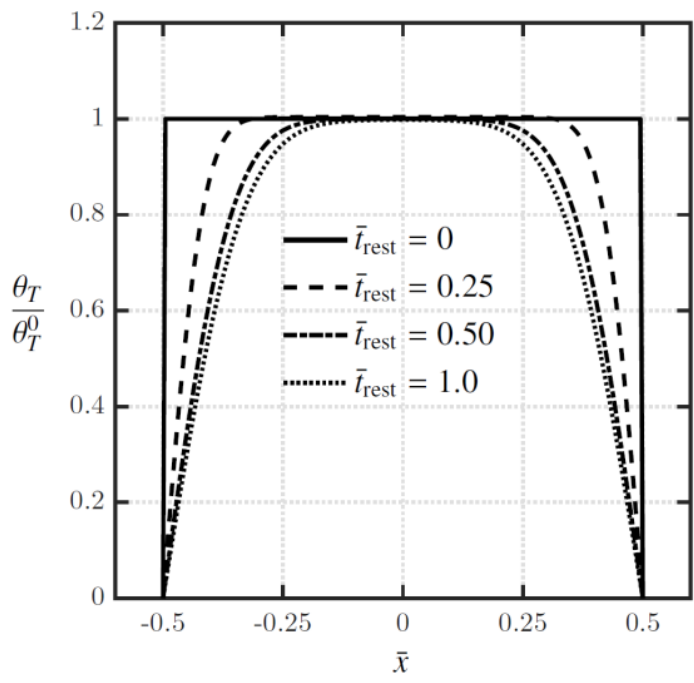

(a)

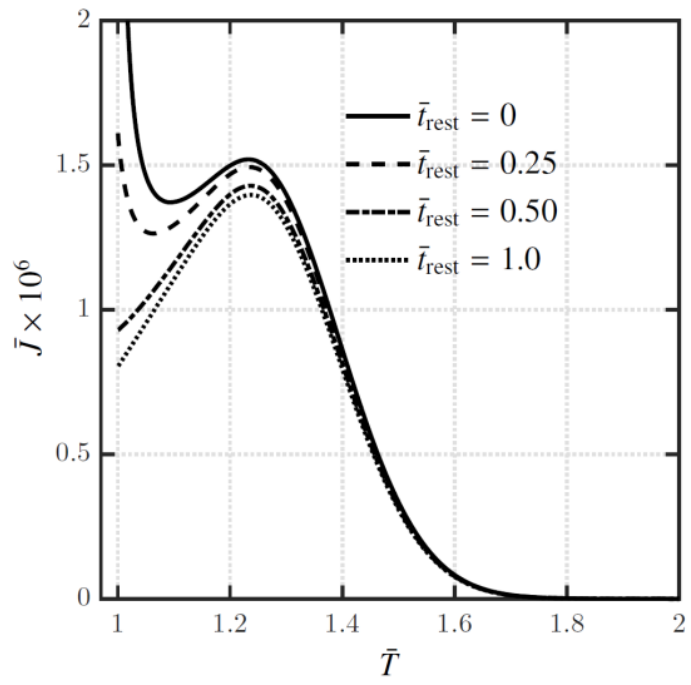

(b)

Figure 6: (a) Spatial distribution of the trap occupancy fraction $\theta_{T}$, normalised by the initial value $\theta_{T}^{0}$, along the specimen thickness $-1 / 2 \leq \bar{x} \leq 1 / 2$ at different rest times $\bar{t}_{\text {rest }}$ for regime I with $\overline{\Delta H}=-8$ (b) TDS simulation with varying rest time $\bar{t}_{\text {rest }}$. Flux $\bar{J}$ is plotted against the temperature $\bar{T}$ in (30) for trap binding energy $\overline{\Delta H}=-8$ The maximum flux and the initial spike decreases with increasing rest time. 